Yu J, Vodyanik MA, Smuga-Otto K, AntosiewiczBourget J, Frane JL, Tian S et al (2007). Induced pluripotent stem cell lines derived from human somatic cells. Science 318: 1917-1920.

Lepore AC, Rauck B, Dejea C, Pardo AC, Rao MS, Rothstein JD et al (2008). Focal transplantationbased astrocyte replacement is neuroprotective in a model of motor neuron disease. Nat Neurosci 11: 1294-1301.

Neuropsychophamacology Reviews (2011) 36, 370-372; doi: I0.1038/npp.2010.140

\section{fMRI Studies of Reward Processing in Adolescent Depression}

Reward function is increasingly considered to be an important aspect of affective disorders such as depression and bipolar disorder. Conceptual models of affective disorders emphasize disrupted reward function as a neural characteristic of low positive affect in depression, and studies with adults have indicated that brain function in reward-related regions distinguishes those with depression from healthy controls. Based on an interest in adolescence, as both a vulnerable period for the onset of affective disorders and a period of ongoing development of neural reward circuitry, an emerging literature is now addressing reward-related brain function in adolescents with depression. Results have generally paralleled those from adult studies and have indicated value in studying reward function in relation to the etiology, pathophysiology, and treatment of depression in young people.

Functional neuroimaging findings indicate that adolescents with depression (Forbes et al, 2009) and adolescents at risk for depression (eg, Gotlib et al, 2010) exhibit low striatal response to rewarding stimuli, such as money or happy facial expressions. This altered brain function has been observed during the anticipation of reward and the receipt of reward, suggesting that adolescent depression involves changes in both the motivation to obtain reward and the enjoyment of rewards once obtained.
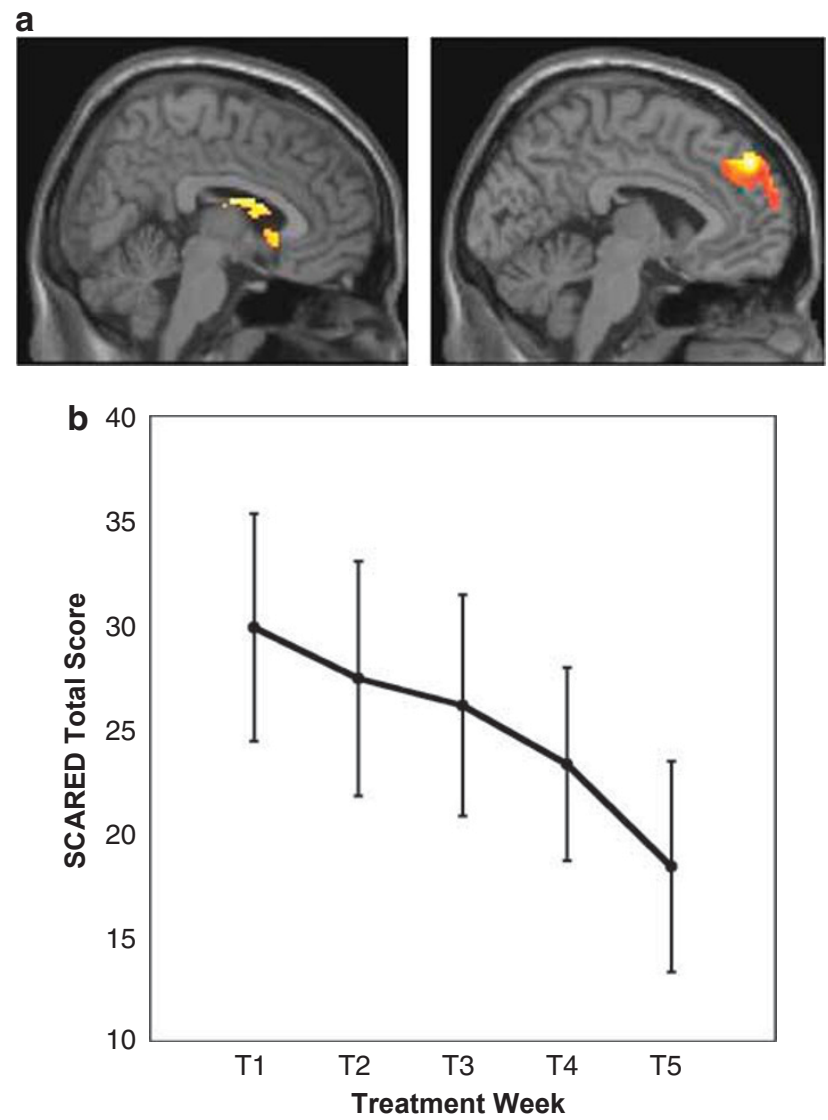

Figure 1. In adolescents with major depressive disorder, (a) response to reward anticipation in the striatum (left) and medial prefrontal cortex (right) at treatment entry was related to (b) rate of reduction in anxiety symptoms during an 8-week treatment with either cognitive-behavioral therapy or cognitive-behavioral therapy plus pharmacotherapy using selective serotonin reuptake inhibitors. Faster rate of decline in anxiety symptoms was predicted by greater striatal reactivity and lower medial prefrontal reactivity before treatment. Error bars represent 1SE of the mean at each time point. SCARED, Screen for Childhood Anxiety and Related Disorders (anxiety symptom measure); T1, pre-treatment; T2, treatment session 2; T3, treatment session 4; T4, treatment session 6; T5, treatment session 8 (post-treatment). The figure is based on the data published in Forbes et al (2010a).

In addition, there is also some evidence that adolescents with depression exhibit more reactivity in prefrontal regions thought to have a role in regulating response to reward stimuli. Consistent with the findings of increased anterior cingulate response to reward in adults with depression, adolescents with depression exhibit less response in the medial prefrontal cortex (PFC) (Forbes et al, 2009), an area that is a key target, along with the striatum, of midbrain dopamine neurons. The medial PFC is a critical region in reward circuits, and its efferent connections include the ventral striatum (Haber and Knutson, 2010).
The study of reward function has potential to elucidate many processes in the development and course of adolescent depression. Developmental findings that reward function changes with puberty and is associated with depressive symptoms in healthy adolescents (Forbes et al, 2010b) can inform the etiology of adolescent depression. Although the treatment implications of reward function research remain to be explored, initial findings suggest that adolescents' neural response to reward predicts symptom level and severity at outcome, as well as rate of symptom decrease during treatment for depression (Forbes et al, 2010a; Figure 1). 
This intriguing result represents one of the few biomarkers of antidepressant response in adolescent depression.

Exciting new approaches to investigating reward function in adolescent depression include examining brainbehavior associations and employing personally relevant social stimuli. When combined with experience sampling, functional neuroimaging can identify regions of the striatum whose response distinguishes adolescents with depression from healthy adolescents and is also correlated with higher levels of positive affect experienced in natural environments (Forbes et al, 2009). Assessing neural response to social rewards, which are postulated to be critical for triggering adolescent depression (Davey et al, 2008), can provide a more meaningful understanding of altered reward function. In addition, future work will benefit from attention to clinical characteristics such as anhedonia, comorbid anxiety and clinical course.

\section{ACKNOWLEDGEMENTS}

This work was supported by K01 MH074769 (PI: Erika E Forbes) and R01 DA026222 (PIs: Erika E Forbes and Daniel S Shaw) from the National Institutes of Health and a NARSAD Young Investigator Award (PI: Erika E Forbes).

\section{Erika E Forbes ${ }^{1}$}

${ }^{1}$ Department of Psychiatry, Western Psychiatric Institute and Clinic, University of Pittsburgh, Pittsburgh, PA, USA

E-mail: forbese@upmc.edu

\section{DISCLOSURE}

The author declares no conflict of interest.

Davey CG, Yücel M, Allen NB (2008). The emergence of depression in adolescence: development of the prefrontal cortex and the representation of reward. Neurosci Biobehav Rev 32: 1-19.

Forbes EE, Hariri AR, Martin SL, Silk JS, Moyles DL, Fisher PM et al (2009). Altered striatal activation predicting real-world positive affect in adolescent major depressive disorder. Am J Psychiatry 166: 64-73.

Forbes EE, Olino TM, Ryan ND, Birmaher B, Axelson D, Moyles DL et al (2010a). Reward-related brain function as a predictor of treatment response in adolescents with major depressive disorder. Cogn Affect Behav Neurosci 10: 107-118.
Forbes EE, Ryan ND, Phillips ML, Manuck SB, Worthman CM, Moyles DL et al (2010b). Healthy adolescents' neural response to reward: associations with puberty, positive affect, and depressive symptoms. J Am Acad Child Adolesc Psychiatry 49: 162-172.

Gotlib $॥ \mathrm{H}$, Hamilton JP, Cooney RE, Singh MK, Henry ML, Joormann J (2010). Neural processing of reward and loss in girls at risk for major depression. Arch Gen Psychiatry 67: 380-387.

Haber SN, Knutson B (2010). The reward circuit: linking primate anatomy and human imaging. Neuropsychopharmacology 35: 4-26.

Neuropsychopharmacology Reviews (2011) 36, 372-373; doi: 10.1038/npp.2010.164

\section{Dorsal vs Ventral Hippocampal Neurogenesis: Implications for Cognition and Mood}

An emerging view of the hippocampus is that of a functionally heterogeneous structure along its longitudinal axis. Lesion studies reveal that the dorsal (septal pole) hippocampus is involved in learning and spatial memory, whereas the ventral (temporal pole) hippocampus regulates emotional and motivated behaviors (Fanselow and Dong, 2010). Anatomical connectivity and gene expression analyses support this functional dissociation. For example, serotonergic fibers provide denser input to the ventral hippocampus with a concomitant enrichment of 5-HT1A and $2 \mathrm{C}$ receptors ventrally (KF Tanaka and $\mathrm{R}$ Hen, unpublished). Efferent connectivity indicates that ventral hippocampus can modulate reward circuitry and emotional behavior through projections to nucleus accumbens, prefrontal cortex and amygdala, and stress responses by regulating the hypothalamic-pituitary-adrenal axis (Sahay and Hen, 2007). In both regions, the subgranular zone of the dentate gyrus (DG) continues to produce new neurons in adulthood. These adult-born granule cells (GCs) functionally integrate into the DG circuit, exhibit enhanced excitability, and have a significant impact on both learning and emotional behavior (Sahay and Hen, 2007). As adult neurogenesis has been implicated in both learning and mood, an exciting possibility is that adult-born GCs in the dorsal and ventral hippocampus may be functionally dissociated.

Chronic antidepressant treatment increases neurogenesis in the DG, a requirement for some of their behavioral effects (Santarelli et al, 2003). Recent studies suggest that antidepressants regulate behavior by selectively increasing ventral hippocampal neurogenesis. Chronic treatment with agomelatine, a melatonin receptor agonist, and 5-HT2C receptor antagonist with robust effects in animal models as well as efficacy in human major depressive disorder increases neurogenesis selectively in the ventral DG (Banasr et al, 2006). In humans, selective serotonin reuptake inhibitors and tricyclic antidepressants increase proliferating and neuronal precursor cells more prominently in the anterior portion of the DG of patients with MDD as compared to controls and untreated MDD subjects (Boldrini et al, 2009). These two studies, although correlational, provide exciting preliminary evidence that warrants future studies aimed at selectively blocking or stimulating neurogenesis in the ventral hippocampus.

In the cognitive realm, adult neurogenesis has recently been implicated in pattern separation, the ability to distinguish between similar contexts (Deng et al, 2010). Owing to their unique physiological properties, adultborn GCs may contribute to pattern separation by modulation of sparse coding in the DG. A recent report indicates that ablation of adult-born GCs increases the magnitude of spontaneous gamma bursts in the dorsal DG and enhances modulation of single unit firing by these bursts (Lacefield et al, 2010). This increase in spontaneous activity suggests that adult-born GCs may modulate network inhibition of mature GCs through either feedback inhibition or synaptic competition. Thus, young GCs may contribute to sparse coding and pattern separation by modulating inhibitory control of the mature GCs in the DG. Deficits 